\title{
The stabilisation of receptor structure in low cross-linked MIPs by an immobilised template $\dagger$
}

\author{
Rosa M. Garcinuño, ${ }^{b}$ Iva Chianella, ${ }^{* a}$ Antonio Guerreiro, ${ }^{a}$ Irene Mijangos, ${ }^{a}$ Elena V. Piletska, ${ }^{a}$ \\ Michael J. Whitcombe ${ }^{a}$ and Sergey A. Piletsky ${ }^{a}$
}

\author{
Received 20th March 2008, Accepted 27th August 2008 \\ First published as an Advance Article on the web 14th October 2008 \\ DOI: $10.1039 / \mathrm{b804476g}$
}

In molecularly imprinted polymers (MIPs) a high level of cross-linking is usually important for preserving the receptor structure. We propose here an alternative approach for stabilising binding sites, which involves the use of an immobilised template. The idea is based on the assumption that an immobilised template will "hold" polymeric chains and complementary functionalities together, preventing the collapsing of the binding sites. To test this postulate, a range of polymers was prepared using polymerisable (2,4-diamino-6-(methacryloyloxy)ethyl-1,3,5-triazine) and non-polymerisable (or extractable) (2,4-diamino-6-methyl-1,3,5-triazine) templates, methacrylic acid as functional monomer and ethylene glycol dimethacrylate as cross-linker. The level of cross-linking was varied from 12 to $80 \%$. Polymerisations were performed in acetonitrile using UV initiation. Binding properties of the synthesised materials were characterised both by HPLC and equilibrium batch binding experiments followed by HPLC-MS or UV-visible detection. The adsorption isotherms of polymers were obtained and fitted to the Langmuir model to calculate dissociation constant, $K_{\mathrm{d}}$, and concentration of binding sites for each material. The results strongly indicate that the presence of an immobilised template improves the affinity of MIPs containing low percentages of cross-linker. The low cross-linked MIPs synthesised with a polymerisable template also retain a reasonable degree of selectivity. Low crosslinked MIPs with such binding characteristics would be useful for the creation of new types of optical and electrochemical sensors, where induced fit or the "gate effect" could be used more effectively for generating and enhancing sensor signals.

\section{Introduction}

In molecularly imprinted polymers a high level of cross-linking seems to be critically important for preserving receptor structure. ${ }^{1-3}$ With very few exceptions, ${ }^{4-6}$ MIPs perform well if the degree of cross-linking is not much lower than $40-50 \%{ }^{7}$ This is actually an advantage for chromatographic applications where highly rigid cross-linked materials are preferred, since they can tolerate high pressures. For sensor applications however the high level of cross-linking is an issue, since the integration of rigid polymers with sensors is not a straightforward process. ${ }^{8}$ In addition to this, rigid polymers are not susceptible to conformational changes, which could be induced by the binding of analytes and used to enhance the sensor response. ${ }^{9,10}$ So what can be done to make MIPs with performances that do not rely on large use of cross-linker? Several years ago we presented one potential solution to this problem: in this work $^{11}$ the problem of fluidity of an imprinted thiol monolayer, formed on a gold surface, was solved by the use of an immobilised template. The

${ }^{a}$ Cranfield Health, Cranfield University, Cranfield, UK MK43 OAL. E-mail: i.chianella.1998@cranfield.ac.uk; Tel: +44(0)1234758322

${ }^{b}$ Dpto. Ciencias Analiticas, Facultad de Ciencias, Universidad Nacional de Educación a Distancia (UNED), Senda del Rey, 9, 28040 Madrid, Spain $\dagger$ Electronic supplementary information (ESI) available: Binding isotherms of $\mathrm{P} 1, \mathrm{P} 4, \mathrm{P} 9$ and $\mathrm{P} 12$ and calculation of binding parameters, Fig. S1-S4. See DOI: 10.1039/b804476g immobilised thiobarbituric acid created sites within a dodecanethiol monolayer which did not disappear as a result of lateral mobility of thiol molecules across the gold surface. These sites were stable and had good affinity and specificity for thiobarbituric and barbituric acids. It was therefore interesting to test if the concept, which had been proven for 2-D MIP layers, could work for 3-D polymers as well. Some indications that it might be possible can be deduced from the work of Baggiani et al., who used immobilised template in MIPs with beneficial effect. $^{12}$ The polymers synthesised in the course of this work, however, contained high levels of cross-linker and it was unclear if the effect would be preserved if cross-linking was significantly reduced. Another reference could be made to an early publication of Hjerten and co-workers, where protein imprinting was performed in gels with relatively low levels of cross-linking. ${ }^{4}$ As reported by Hjerten himself in a personal communication, ${ }^{13}$ it seemed that the complete removal of the template had a detrimental effect on MIP recognition properties. One explanation could be that the remaining template stabilised the receptor structures in the polymer. The investigation of this would have been difficult however, since the template was not covalently linked to the polymer and information about its concentration in the gel was not available. Here we present the first systematic investigation of the use of an immobilised template in MIPs prepared with varying degrees of cross-linking. 


\section{Experimental}

\section{Reagents}

2,4-Diamino-6-methyl-1,3,5-triazine, methacrylic acid (MAA), ethylene glycol dimethacrylate (EGDMA), methyl methacrylate (MMA), 1,1'azobis(cyclohexane carbonitrile), desmetrym, terbumeton, isoproturon and melamine were purchased from Sigma-Aldrich (Dorset, UK). 2,4-Diamino-6-(methacryloyloxy)ethyl-1,3,5-triazine was purchased from Wako (Japan). Acetonitrile and acetone of HPLC grade were purchased from Acros Organics (Fisher Scientific UK, Loughborough, UK). All reagents and solvents were of analytical grade and used as received.

\section{Preparation of molecularly imprinted polymers}

The template molecules: 2,4-diamino-6-methyl-1,3,5-triazine (template, T), 2,4-diamino-6-(methacryloyloxy)ethyl-1,3,5triazine (polymerisable template analogue, $\mathrm{pT}$ ) or a mixture of the two, was placed in a screw-cap vial and dissolved in acetonitrile. The functional monomer (methacrylic acid, MAA) was then added, followed by a calculated amount of cross-linker: EGDMA or its mixture with methyl methacrylate, equal to around $80 \%(\mathrm{w} / \mathrm{w})$ of the total polymer mixture. The composition of the polymers is described in Table 1 and Table 2. For the preparation of polymers P5 and P6 the monomer, MAA, was replaced by the same molar quantity of acetic acid to obtain representative controls. The initiator, 1, $1^{\prime}$-azobis(cyclohexane carbonitrile), was added at an amount equivalent to $2 \%$ of the total weight of polymerisation mixture. The solutions were purged with nitrogen and the vials were sealed. Polymerisation was performed under a UV lamp (UVAPRINT 100 CVI UV source with $0.163 \mathrm{~W} \mathrm{~cm}^{-2}$ intensity, purchased from Dr Hönle AG, Germany) for 15 minutes. The resulted bulk polymers were broken with a steel spatula, manually ground in methanol using a mortar and wet-sieved to collect polymer particles in the size range $25-106 \mu \mathrm{m}$. The fine particles were removed by repeated suspension in methanol, sedimentation and decanting of the supernatant. The collected particles were stored in a solvent for further study. Corresponding non-imprinted polymers (NIPs) were made in the absence of template and were prepared and

Table 1 Composition of the synthesised polymers ${ }^{d}$

\begin{tabular}{|c|c|c|c|c|}
\hline \multirow[b]{2}{*}{ Polymer } & \multicolumn{4}{|c|}{$\begin{array}{l}\text { Amount } / \mathrm{mmol} \text { (amounts in grams are for the synthesis of } \\
\sim 2.65 \mathrm{~g} \text { of polymer) }\end{array}$} \\
\hline & $\mathrm{T}^{a}$ & $\mathrm{pT}^{b}$ & $\begin{array}{l}\text { Monomer } \\
\text { (MAA) }\end{array}$ & $\begin{array}{l}\text { Cross-linker } \\
\text { (EGDMA) }\end{array}$ \\
\hline $\mathrm{P} 1$ & $1.00(0.084 \mathrm{~g})$ & 0.00 & $8.63(0.500 \mathrm{~g})$ & $15.01(2.00 \mathrm{~g})$ \\
\hline $\mathrm{P} 2$ & $0.90(0.076 \mathrm{~g})$ & $0.10(0.015 \mathrm{~g})$ & $8.63(0.500 \mathrm{~g})$ & $15.01(2.00 \mathrm{~g})$ \\
\hline P3 & $0.50(0.042 \mathrm{~g})$ & $0.50(0.075 \mathrm{~g})$ & $8.63(0.500 \mathrm{~g})$ & $15.01(2.00 \mathrm{~g})$ \\
\hline P4 & 0.00 & $1.00(0.150 \mathrm{~g})$ & $8.63(0.500 \mathrm{~g})$ & $15.01(2.00 \mathrm{~g})$ \\
\hline P5 & 0.00 & $1.00(0.150 \mathrm{~g})$ & $0.00^{c}$ & $15.01(2.00 \mathrm{~g})$ \\
\hline P6 & $1.00(0.084 \mathrm{~g})$ & 0.00 & $0.00^{c}$ & $15.01(2.00 \mathrm{~g})$ \\
\hline $\mathrm{C} 1$ & 0.00 & 0.00 & $8.63(0.500 \mathrm{~g})$ & $15.01(2.00 \mathrm{~g})$ \\
\hline
\end{tabular}

treated in the same manner as their imprinted counterparts. The methanol washings deriving from grinding the polymers made with pT were collected and analysed by HPLC-MS to assess the presence of non-polymerised $\mathrm{pT}$. The quantification of released pT was performed using a Waters HPLC in tandem with a bench-top triple quadrupole mass spectrometer (Micromass Quattro Micro, Waters, UK) equipped with an electrospray probe. The values of the voltages applied to the sampling cone $(20 \mathrm{~V})$, capillary $(3.02 \mathrm{~V})$, extractor $(2 \mathrm{~V})$ and collision cell $(2 \mathrm{mV})$ were optimised by continuous infusion in order to achieve the highest possible sensitivity. HPLC-MS analyses of pT were carried out in SIR mode, where the parent molecule $(\mathrm{m} / \mathrm{z} 224)$ was monitored. The electrospray probe was maintained at $+350{ }^{\circ} \mathrm{C}$ with a spray voltage of $450 \mathrm{~V}$ for positive ionisation mode. The electron multiplier was set at $650 \mathrm{~V}$. For the HPLC conditions $80 \%$ acetonitrile containing $0.1 \%$ formic acid was used as mobile phase with a flow rate of $0.2 \mathrm{ml} \mathrm{min}^{-1}$ and the injection volume was $10 \mu \mathrm{L}$. The column, which was a Luna 18 $(3 \mu \mathrm{m}, 3 \mathrm{~mm} \times 50 \mathrm{~mm}$ ) from Phenomenex (Macclesfield, UK) was kept at $40{ }^{\circ} \mathrm{C}$. The quantification was performed using the MassLynx software by monitoring the peak of $\mathrm{pT}$, which had a retention time of $1.73 \mathrm{~min}$.

\section{Chromatographic evaluation}

An adequate amount of polymer particles suspended in methanol or acetonitrile were packed into stainless steel HPLC columns $(150 \times 4.6 \mathrm{~mm})$ at a constant pressure of 2000 psi using a 1666 Slurry Packer (Alltech, Lancashire, UK). Chromatographic measurements were performed using an HPLC system, which included a 325-Kronon Instruments solvents delivery system, a Kronon Instruments autosampler (UK), and a Bio-Tek Instruments model 535 HPLC detector (Vermont, USA). The packed columns were first washed on-line with acetonitrile-water (85: 15) at a constant flow rate of $1.5 \mathrm{~mL} \mathrm{~min}^{-1}$ until a stable baseline was reached to ensure the removal of template, which could still have been entrapped inside the polymer after the synthesis. Subsequent chromatographic analysis was executed with the same mobile phase and at the same flow rate $(1.5 \mathrm{~mL}$ $\left.\mathrm{min}^{-1}\right)$. The template concentration and injection volume were $1 \mathrm{mg} \mathrm{mL}^{-1}$ and $20 \mu \mathrm{L}$, respectively. Each elution was repeated at least three times to assure reproducibility of the chromatograms. The analysis was performed using UV detection at $250 \mathrm{~nm}$. Column void volumes were measured by injection of $20 \mu \mathrm{L}$ of acetone $(0.01 \%, \mathrm{v} / \mathrm{v})$ in the corresponding mobile phase. Capacity factors $\left(k^{\prime}\right)$ were calculated as $\left(t_{\mathrm{T}}-t_{\mathrm{o}}\right) / t_{\mathrm{o}}$, where $t_{\mathrm{T}}$ is the retention time of the eluted substance and $t_{\mathrm{o}}$ the retention time of the void marker (acetone). The imprinting factor ( $I$ ) was calculated as $I=k_{\text {MIP }}^{\prime} / k_{\text {NIP }}^{\prime}$, where $k_{\text {MIP }}^{\prime}$ is the capacity factor of the template molecule eluted from the imprinted polymer and $k_{\text {NIP }}^{\prime}$ is the capacity factor of the template molecule eluted from the non-imprinted polymer.

\section{Batch binding experiments for adsorption isotherms}

Equilibrium batch binding experiments were performed to obtain the polymer adsorption isotherms and calculate binding parameters. Briefly, a small amount $(20 \mathrm{mg})$ of high $(80 \%)$ and low $(12 \%)$ cross-linked MIPs synthesised with polymerisable and 
Table 2 Composition of polymers synthesised with different amount of cross-linker ${ }^{c}$

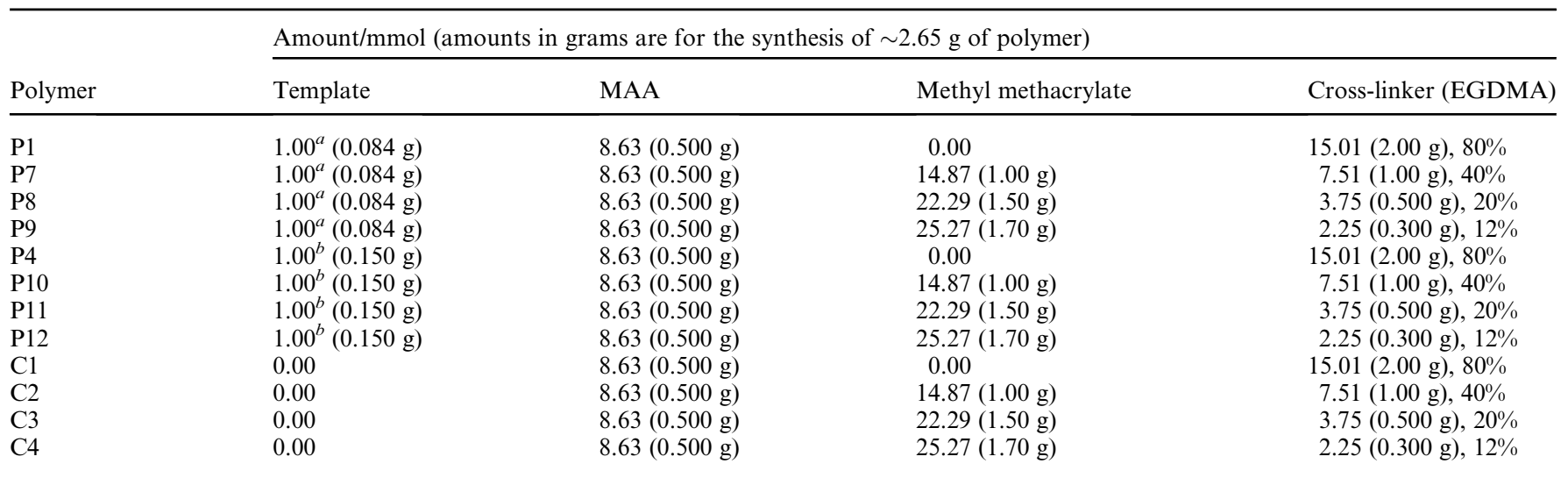

${ }^{a}$ 2,4-Diamino-6-methyl-1,3,5-triazine. ${ }^{b}$ 2,4-Diamino-6-(methacryloyloxy)ethyl-1,3,5-triazine. ${ }^{c}$ The composition also contained $2.68 \mathrm{~g}$ of acetonitrile and $0.054 \mathrm{~g}\left(2 \% \mathrm{w} / \mathrm{w}\right.$ of total) of initiator $1,1^{\prime}$ azobis (cyclohexane-carbonitrile).

extractable template (P1, P4, P9 and P12; see Table 2 for polymer compositions) was incubated and mechanically shaken

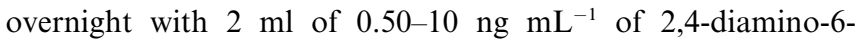
methyl-1,3,5-triazine (template, $\mathrm{T}$ ) prepared in acetonitrilewater $(85: 15)$. After removal of the polymer particles by filtration, quantification of free $\mathrm{T}$ (and by subtraction the adsorbed $\mathrm{T}$ ) was carried out by analysis of the solutions via HPLC-MS. For quantification of $\mathrm{T}$ concentration, the values of the voltages applied to the sampling cone $(25 \mathrm{~V})$, capillary $(4.5 \mathrm{~V})$, extractor $(2 \mathrm{~V})$ and collision cell $(2 \mathrm{mV})$ were optimised by continuous infusion in order to achieve the highest possible sensitivity. HPLC-MS analyses of T were carried out in SIR mode, where the parent molecule $(\mathrm{m} / \mathrm{z} 126)$ was monitored. The electrospray probe was maintained at $+350{ }^{\circ} \mathrm{C}$ with a spray voltage of $450 \mathrm{~V}$ for positive ionisation mode. The electron multiplier was set at $650 \mathrm{~V}$. The HPLC conditions were the same as for the analysis of $\mathrm{pT}$, which was described above. The quantification was performed using MassLynx software through a calibration curve built with standard solutions of template. The peak of the template with a retention time of 1.47 min was monitored. The binding parameters ( $K_{\mathrm{d}}$ and binding capacity) were estimated by curve fitting using the Grafit package, Erithacus Software. ${ }^{14}$

\section{Evaluation of polymer selectivity by batch binding experiments}

The selectivity of low cross-linked (12\%) MIPs made with extractable and polymerisable template (P9 and P12) and the low cross-linked (12\%) control NIP (C4) was assessed by equilibrium batch binding experiments. The binding of the polymers to several analogues of the template such as desmetrym, terbumeton, pT and melamine was investigated. The binding to a nonstructurally related compound, isoproturon, was also studied. The structures of all the tested compounds are reported in Fig. 1. The experiments were carried out by overnight incubation of $25 \mathrm{mg}$ of each polymer in Zeba spin-cartridges (purchased from Pierce, Perbio Science, UK) with $500 \mu \mathrm{l}$ of $0.1 \mathrm{mg} \mathrm{mL}^{-1}$ of each compounds (including the template), prepared in acetonitrile-water $85: 15$. The experiments were performed in triplicates. After incubation the spin-cartridges were centrifuged at

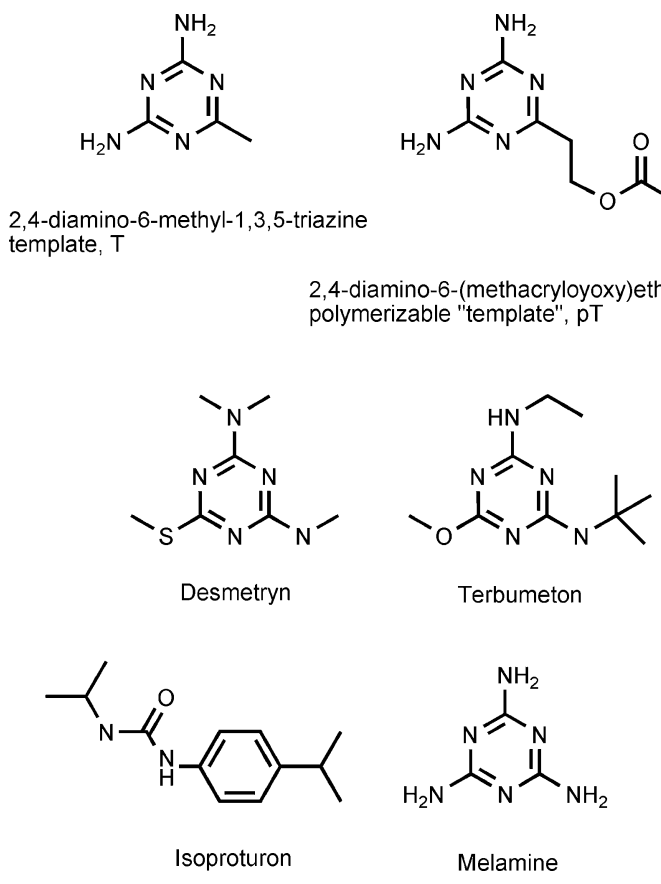

Fig. 1 Structures of the free (extractable) and polymerisable templates and of the analogues tested for cross-reactivity.

$6500 \mathrm{rpm}$ for $30 \mathrm{~s}$ to separate the supernatant. The solutions were then collected and the concentrations of analytes quantified by HPLC using a Gemini $\mathrm{C}_{18}(5 \mu \mathrm{m}, 150 \mathrm{~mm} \times 2 \mathrm{~mm})$ from Phenomenex. Chromatographic analysis was performed using acetonitrile-water $(85: 15)$ as the mobile phase at a flow rate of $0.5 \mathrm{~mL} \mathrm{~min}{ }^{-1}$. The injection volume of all the tested compounds was $20 \mu \mathrm{L}$. Each injection was repeated at least twice to assure reproducibility of the chromatograms. The analysis was performed using UV detection at $250 \mathrm{~nm}$, except for melamine which was analysed at $240 \mathrm{~nm}$.

\section{Swelling analysis}

Swelling experiments were performed using $200 \mathrm{mg}$ of polymer particles $(25-106 \mu \mathrm{m})$ packed in $1 \mathrm{~mL}$ graduated syringe 
cartridges (Supelco, UK). The cartridges were filled with $0.5 \mathrm{~mL}$ of acetonitrile. After equilibration at room temperature, the excess of solvent was removed from the polymer by the brief application of reduced pressure $(1 \mathrm{~min})$ and the volume of the swollen polymer was measured. The swelling ratio $(\mathrm{Sr})$ of the polymers was calculated from the following equation: $\mathrm{Sr}=$ $\left(V_{\mathrm{s}}-V_{\mathrm{o}}\right) / V_{\mathrm{o}}$, where $V_{\mathrm{s}}$ is the volume of the swollen polymer and $V_{\mathrm{o}}$ is the volume of the dry polymer.

\section{Results and discussion}

Two triazine compounds were selected as templates for the model study: 2,4-diamino-6-methyl-1,3,5-triazine (T) and 2,4diamino-6-(methacryloyloxy) ethyl-1,3,5-triazine (pT) (see Fig. 1). The polymerisable template analogue contains a methacryloyl residue, which can be used for its immobilisation into a polymeric network. For a matter of simplicity, this polymerisable analogue will be addressed as 'immobilised or polymerisable template, $\mathrm{pT}$ ' even if it does not create imprints like a classic template for molecular imprinting. In the initial phase of experiments several polymers were synthesised containing a fixed quantity of methacrylic acid but using different ratios between the polymerisable and non-polymerisable (or extractable) templates. One polymer was prepared in the absence of any template as a control (C1) and two others (P5 and P6) were made in the absence of methacrylic acid. The compositions of polymers synthesised are given in Table 1 . The affinities of the polymers for template were determined by HPLC. The mobile phase selected for this was acetonitrile-water $(85: 15)$, which allowed the polymer affinity to be rapidly analysed. Binding in water or in pure acetonitrile was too strong, as a result of non-specific hydrophobic or electrostatic interactions. The results of testing are presented in Table 3 . The HPLC data clearly proves that the presence of the extractable template in the monomer mixture substantially enhanced polymer affinity. The imprinting factor (comparing results for MIPs and control polymers) was as high as 28.7 (for P1).

Replacing the extractable template with a polymerisable template led to a gradual decrease in the measured capacity factor. A similar trend was observed when the polymerisable template was injected (data not shown). Since P4, made with immobilised template, still had a small imprinting factor of 1.34, it can be concluded that the immobilised template plays some

Table 3 Chromatographic performances of the polymers synthesised with different ratios of extractable and polymerisable template

\begin{tabular}{lcll}
\hline Polymer & $\begin{array}{c}\text { Molar ratio } \\
\mathrm{T}: \mathrm{pT}\end{array}$ & $\begin{array}{l}\text { Capacity factor }^{a} \\
k^{\prime}=\left(t_{\mathrm{T}}-t_{\mathrm{o}}\right) / t_{\mathrm{o}}\end{array}$ & $\begin{array}{l}\text { Imprinting factor }^{a} \\
I=k^{\prime}(\mathrm{MIP}) / k^{\prime}(\mathrm{NIP})\end{array}$ \\
\hline P1 & $100: 0$ & 68.50 & 28.66 \\
P2 & $90: 10$ & 56.00 & 23.43 \\
P3 & $50: 50$ & 16.50 & 6.90 \\
P4 & $0: 100$ & 3.20 & 1.34 \\
P5 & $0: 100$ & 0.2 & - \\
P6 & $100: 0$ & - & - \\
C1 & $0: 0$ & 2.39 & -
\end{tabular}

${ }^{a}$ Flow rate: $1.5 \mathrm{~mL} \mathrm{~min}^{-1}$, mobile phase: acetonitrile-water $(85: 15)$. Injection amounts of template were $1 \mathrm{mg} \mathrm{mL}^{-1}$ in a $20 \mu \mathrm{l}$ injection volume. All measurements were made in triplicate. The STD in all tests was below $5 \%$. positive role in molecular recognition e.g. through formation of a minimal amount of template-template complexes or molecular crystallisation. This would be in agreement with the work by Allender and co-workers, who have reported presence of template-template interactions for a triazine compound (atrazine) when testing was performed in $100 \%$ acetonitrile. ${ }^{15}$ In our study, very little or no binding was, however, observed to polymers made without MAA using either pT or T (P5 and P6). This clearly indicates that in our experimental conditions template (T) does not form stable complexes with polymerisable template $(\mathrm{pT})$.

In the second phase of our work we set out to investigate whether it would be possible to preserve MIP binding sites, at least partially, by decreasing the level of cross-linking and consequently increasing the polymer swelling capabilities.

The assumption was that a polymerisable template could assist in preserving binding sites through three possible mechanisms, schematically presented in Fig. 2. According to the first, the polymerisable template can contribute to the creation of specific imprinted binding sites in the same way as non-polymerisable template does. The swelling process pulls some polymer chains apart, separating template moieties from their complementary functional groups within their respective binding cavities (Fig. 2A). As a result, the binding sites become temporarily vacant and can be occupied either by free template or once again by immobilised template molecules. The proximity of the immobilised template would help to preserve the structure of the polymer by the reversible nature of the dissociation between polymer and polymerised template, preventing the complete separation of neighbouring chains and loss of polymer affinity. The second mechanism relies upon stabilisation of the orientation of polymer chains through the template-monomer interactions (Fig. 2B). In essence, the template plays the role of physical cross-linker, which prevents uncontrolled diffusion of polymer chains. Another possible mechanism, which needs to be taken into consideration, is the one described in Fig. 2C. In this case, the immobilised template can act as a receptor by binding free template through formation of template-template complexes or molecular crystallisation. As already explained above, in the tested conditions, template-template complexes do not seem to take place in highly cross-linked MIPs prepared with pT (see testing of P5). On the other hand, as the cross-linking is decreased the polymer swelling and therefore the availability of pT would increase and this could enhance the probability to have template-template interactions. Therefore a combination of the three mechanisms in which pT helps in preserving binding sites and at the same time behaves both as a physical cross-linker and partially as a receptor could be the most plausible.

The feasibility of the proposed mechanisms was therefore studied by synthesis and investigation of polymers with reduced amounts of cross-linker. To compensate for changes in mass, the "missing" cross-linker was replaced with "inert" methyl methacrylate. The polymer compositions are presented in Table 2 .

The synthesised polymers were tested chromatographically as described above. The results of testing show that for "normal" MIPs, synthesised using the extractable template, a reduction in the level of cross-linking leads to a massive decrease in polymer affinity for template (Table 4). It seems that imprinting does not 

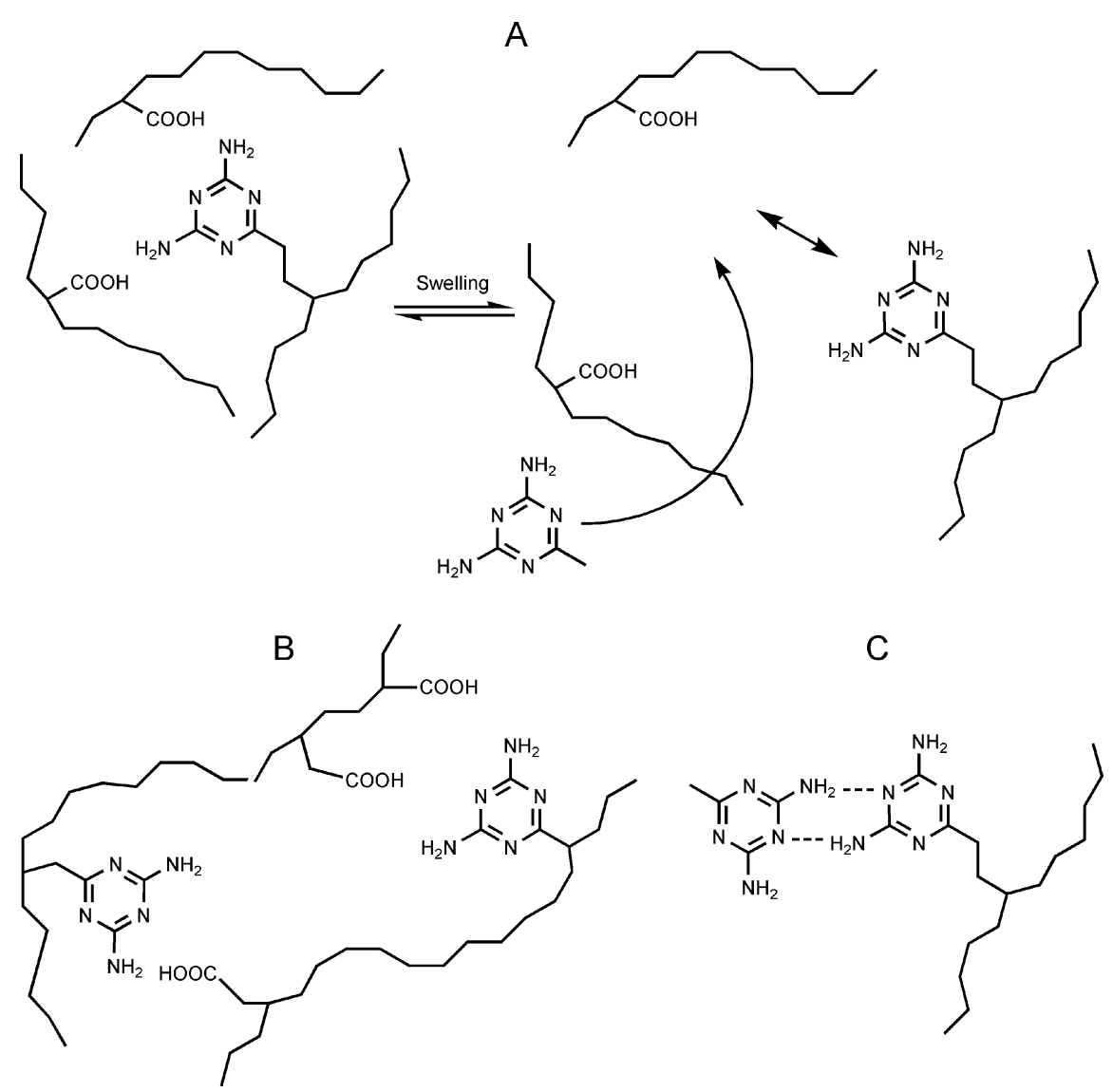

Fig. 2 Proposed mechanisms contributing towards stabilisation of the orientation of polymer chains in the materials prepared using polymerisable template.

Table 4 Chromatographic performances of the polymers synthesised with or without extractable template $(\mathrm{T})$ and with varying amounts of cross-linker

\begin{tabular}{llll}
\hline Polymer & Cross-linker $(\%)$ & $\begin{array}{l}\text { Capacity factor }^{a} \\
k^{\prime}=\left(t_{\mathrm{T}}-t_{\mathrm{o}}\right) / t_{\mathrm{o}}\end{array}$ & $\begin{array}{l}\text { Imprinting factor }^{a} \\
I=k^{\prime}(\mathrm{MIP}) / k^{\prime}(\mathrm{NIP})\end{array}$ \\
\hline P1 & \multirow{2}{*}{80} & 68.50 & 28.66 \\
C1 & & 2.39 & \\
P7 & \multirow{2}{*}{40} & 6.41 & 2.43 \\
C2 & & 2.64 & \\
P8 & 20 & 3.69 & 1.54 \\
C3 & \multirow{2}{*}{12} & 2.39 & \\
P9 & & 1.30 & 0.5 \\
C4 & & 2.48 &
\end{tabular}

${ }^{a}$ Flow rate: $1.5 \mathrm{~mL} \min ^{-1}$, mobile phase: acetonitrile-water $(85: 15)$. Injection amounts of template were $1 \mathrm{mg} \mathrm{mL}^{-1}$ in a $20 \mu \mathrm{l}$ injection volume. All measurements were made in triplicate. The STD in all tests was below $5 \%$.

work when the concentration of cross-linker approaches $12 \%$ This was entirely as expected. However, the results for polymers prepared using the immobilised template were very different (Table 5). In this case it can be seen that even if the imprinting factor was never very high, it was definitely increasing as the cross-linking was decreased. This tendency is opposite to that observed for MIPs prepared using normal, extractable template.
Table 5 Chromatographic performances of the polymers synthesised with or without polymerisable template (pT) and with varying amounts of cross-linker

\begin{tabular}{llll}
\hline Polymer & Cross-linker $(\%)$ & $\begin{array}{l}\text { Capacity factor }^{a} \\
k^{\prime}=\left(t_{\mathrm{T}}-t_{\mathrm{o}}\right) / t_{\mathrm{o}}\end{array}$ & $\begin{array}{l}\text { Imprinting factor }^{a} \\
I=k^{\prime}(\mathrm{MIP}) / k^{\prime}(\mathrm{NIP})\end{array}$ \\
\hline P4 & \multirow{2}{*}{80} & 3.20 & 1.34 \\
C1 & & 2.39 & \\
P10 & 40 & 5.00 & 1.89 \\
C2 & & 2.64 & \\
P11 & 20 & 4.54 & 1.90 \\
C3 & \multirow{2}{*}{12} & 2.39 & \\
P12 & & 4.98 & 2.01 \\
C4 & & 2.48 &
\end{tabular}

${ }^{a}$ Flow rate: $1.5 \mathrm{~mL} \mathrm{~min}^{-1}$; mobile phase: acetonitrile-water $(85: 15)$. Injection amounts of template were $1 \mathrm{mg} \mathrm{mL}^{-1}$ in a $20 \mu$ injection volume. All measurements were made in triplicate. The STD in all tests was below $5 \%$.

Similar results were also obtained from batch binding experiments performed with high $(80 \%)$ and low $(12 \%)$ cross-linked MIPs synthesised in the presence of either T or pT (P1, P9, P4 and P12). Dissociation constants, $K_{\mathrm{d}}$, and binding capacity (concentration of binding sites) obtained by fitting the binding curves to the Langmuir model are reported in Table 6 .

The binding curves of the four polymers and the fittings parameters are available in the ESI† (see Fig. S1, S2, S3 and S4). 
Table 6 Dissociation constants and concentration of binding sites calculated by fitting the binding curves to a one-site Langmuir model ${ }^{a}{ }^{14}$

\begin{tabular}{lll}
\hline Polymer & $K_{\mathrm{d}} / \mathrm{M}$ & Binding sites/M \\
\hline $\mathrm{P} 1,80 \%, \mathrm{~T}$ & $1.00 \times 10^{-5} \pm 0.18 \times 10^{-5}$ & $9.94 \times 10^{-5} \pm 0.86 \times 10^{-5}$ \\
$\mathrm{P} 4,80 \%, \mathrm{pT}$ & $1.50 \times 10^{-5} \pm 0.33 \times 10^{-5}$ & $2.36 \times 10^{-5} \pm 0.18 \times 10^{-5}$ \\
$\mathrm{P} 9,12 \%, \mathrm{~T}$ & $2.01 \times 10^{-5} \pm 0.43 \times 10^{-5}$ & $2.43 \times 10^{-5} \pm 0.34 \times 10^{-5}$ \\
$\mathrm{P} 12,12 \%, \mathrm{pT}$ & $1.24 \times 10^{-5} \pm 0.34 \times 10^{-5}$ & $1.90 \times 10^{-5} \pm 0.17 \times 10^{-5}$ \\
${ }^{a}$ The one site Langmuir model was selected since under the used \\
experimental conditions, only one population of binding sites were \\
found to contribute to template binding.
\end{tabular}

The one-site Langmuir model was selected since under the experimental conditions used, only one population of binding sites were found to contribute to template binding. Table 6 shows that P1, which is the MIP prepared with $80 \%$ cross-linking and extractable template $(\mathrm{T})$, possessed the highest affinity and the highest concentration of binding sites. The affinity and capacity of $\mathrm{P} 1$ for the template $\mathrm{T}$ was so high that a total binding of the smaller concentrations tested for the experiments was obtained producing a loss of data points (see Fig. S1, ESI $\dagger$ ). However, for comparison reasons the testing conditions for P1 were kept as for the other three polymers. P9, which is the MIP synthesised with $12 \%$ of cross-linker and extractable template had the worst affinity for T, proving again that low cross-linked MIPs prepared with 'normal' templates are not ideal for analytical applications. On the other hand, P4 and P12, which are MIPs prepared with polymerisable template (pT) and $80 \%$ and $12 \%$ of cross-linker, respectively, seemed to show the opposite behaviour. In fact, P12, the low cross-linked MIP, possessed higher affinity for the template than $\mathrm{P} 4$, even though the latter appeared to have a higher capacity. These results are in good agreement with the mechanisms reported in Fig. 2A and B, where pT, when present in the polymer structure, helps in preserving the binding sites in low-cross linked material (affinity of P12 is higher than that of P4). At the same time, part of $\mathrm{pT}$ might be also acting as a physical cross-linker helping in maintaining the orientation of the polymer functionalities without producing active binding sites (concentration of binding sites of P4 is higher than that of P12). This latter observation seems also to disprove the third mechanism (see Fig. 2C), which reports template-template interactions in swollen low cross-linked materials. In fact, if this was happening also higher binding capacity and not only affinity should be observed for P12. Baggiani and co-workers have reported that in their testing system template-template interactions seemed to be mainly responsible for the recognition of the template by an immobilised analogue. ${ }^{12}$ Therefore to further investigate (and possibly further disprove) whether this might be the case with our system, we have analysed the dependence of P12 capacity factor on the concentration of template in solution. The expectation was that if the template forms clusters around the immobilised template, then binding should increase upon an increase in template concentration. On the contrary, however, the results of this study look very similar to the plots usually observed for traditional MIPs (see Fig. 3). Thus although it would be impossible to deny that the template under some different testing conditions can interact with the immobilised template analogue, ${ }^{15}$ it was not shown to be a major factor

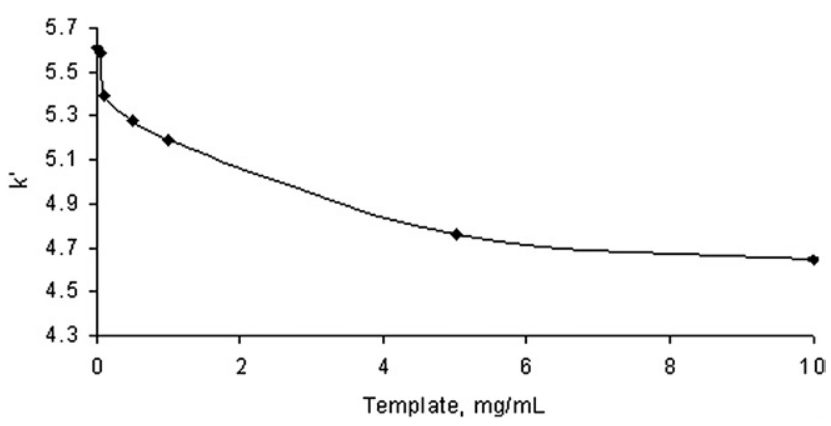

Fig. 3 Dependence of the capacity factor on the concentration of template in solution. HPLC conditions: flow-rate $0.8 \mathrm{~mL} \mathrm{~min}{ }^{-1}$; acetonitrile-water $(85: 15)$ as mobile phase. Injection amounts were $0.01-1$ $\mathrm{mg} \mathrm{mL}^{-1}$ in a $20 \mu \mathrm{l}$ injection volume. All measurements were made in triplicate. The STD in all tests was below $5 \%$.

involved in the recognition process in our study. There is also no evidence that part of non-polymerised pT could act as "normal" template since polymeric extracts, analysed by HPLC-MS, did not contain traces of this molecule. Practically the entire amount of pT present in the monomer mixtures was therefore successfully integrated into the polymers.

We have attempted to find further proof of the binding mechanisms described in Fig. 2 by measuring the effect of the template on polymer swelling. The result of this study, which is reported in Fig. 4, confirms that the first two mechanisms are indeed plausible. One direct conclusion from these data is that the immobilised template reduces polymer swelling by acting as an equivalent of the cross-linker. Furthermore the addition of template to the acetonitrile increased polymer swelling by inserting among the polymeric chains pushing them apart, which is in agreement with the models proposed in Fig. 2A and B. Thus, our current understanding is that the first two mechanisms might be the most plausible and possibly are complementing each other; the specific binding sites would be created by association and dissociation of template-monomer complexes as result of polymer chains fluctuations. These chains would remain however

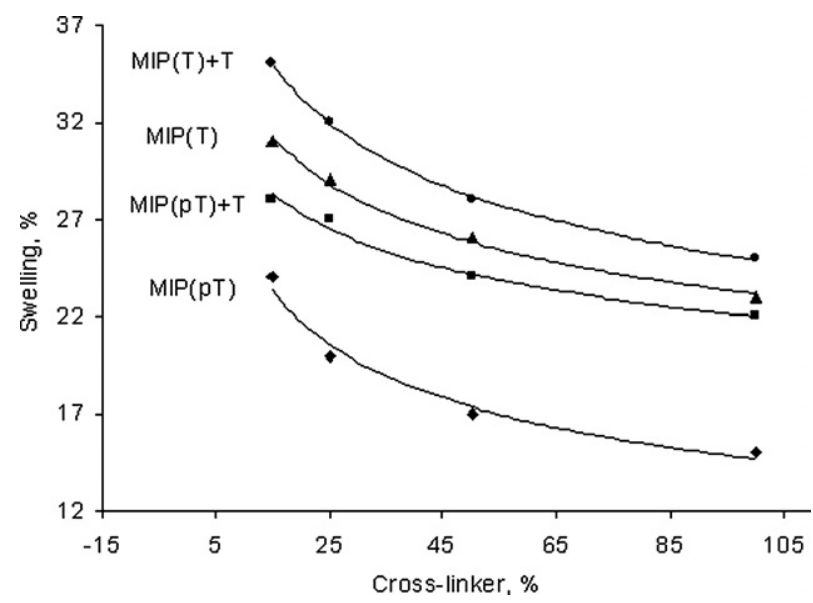

Fig. 4 Polymer swelling in acetonitrile in the presence and absence of template: $\mathrm{MIP}(\mathrm{T})$ are polymers made in the presence of extractable template. $\operatorname{MIP}(\mathrm{pT})$ are polymers made in the presence of polymerisable template. MIP(T) + T and MIP $(\mathrm{pT})+\mathrm{T}$ indicate swelling tests performed in the presence of $1 \mathrm{mg} \mathrm{mL}^{-1}$ of template. 
Table 7 P9, P12 and C4 specificity evaluated by equilibrium batch binding experiments ${ }^{a}$

\begin{tabular}{|c|c|c|c|c|c|c|}
\hline Polymer & \multicolumn{6}{|c|}{ Amount $(\mu \mathrm{g})$ of adsorbed compound per $25 \mathrm{mg}$ of polymer } \\
\hline P12 & $26.3 \pm 1.6$ & $14.1 \pm 0.5$ & $9.1 \pm 3.3$ & $4.5 \pm 0.6$ & $4.5 \pm 2.2$ & $0 \pm 0.2$ \\
\hline $\mathrm{C} 4$ & $19.5 \pm 3.0$ & $18.1 \pm 0.1$ & $38.6 \pm 2.4$ & $5.7 \pm 0.3$ & $6.1 \pm 0.4$ & $3.4 \pm 2.8$ \\
\hline
\end{tabular}

${ }^{a}$ The experiments were performed by overnight incubation of $500 \mu \mathrm{L}$ of $0.1 \mathrm{mg} \mathrm{mL}^{-1}$ of each compound with $25 \mathrm{mg}$ of each polymer. The experiments were repeated in triplicate.

in close proximity to each other due to partial (physical) crosslinking provided by monomer-template interactions.

The final study performed was an evaluation of the selectivity of low cross-linked polymers. The selectivity of lightly crosslinked polymers (12\% cross-linking) P9, P12 and C4 was investigated with five different template analogues (see Fig. 1) in batch binding experiments. Template $(\mathrm{T})$, two triazine herbicides (desmetryn and terbumeton), pT, a small triazine (melamine) and a non-triazine herbicide isoproturon, were selected for this study. The results of the experiments are reported in Table 7. P12 shows selective binding to $\mathrm{T}$ over all of the analogues, with the next highest binding to $\mathrm{pT}$, thus demonstrating the highest selectivity for the template among the polymers tested. P9 shows both worse specificity and selectivity. This selectivity study therefore suggests once again that the presence of the immobilised template, $\mathrm{pT}$ helps to maintain the orientation of the functional groups in the binding cavities, not just improving the affinity, but also the specificity of the resulting polymers.

\section{Conclusions}

In conclusion, we have reported here that the presence of an immobilised template has a positive effect on molecular recognition in lightly cross-linked imprinted polymers. MIPs synthesised using a polymerisable template analogue $(\mathrm{pT})$ retained affinity and specificity for free template $(\mathrm{T})$ even when prepared with a level of cross-linker as low as $12 \%$. According to the experiments carried out, this is probably due to a double role of the immobilised template, which helps in preserving MIPs binding cavities in low cross-linked materials both by maintaining interactions with the binding sites functionalities and at the same time by partially acting as a physical cross-linker. In addition more than one experiment pointed out that under our testing conditions template-template interactions between $\mathrm{pT}$ and $\mathrm{T}$ do not appear to have a major contribution to the recognition capability of the low cross-linked MIPs.
One interesting opportunity offered by these findings lies in the possibility of designing intelligent detection systems where fluorescent analogues can be displaced by template from the binding sites. The variations in fluorescence as a result of a change in the environment of the fluorophore can be used for quantification of template in solution. Enhanced polymer swelling by the template could also be used in the future for the development of new sensors. Detection in this case could be achieved by electrochemical ${ }^{16}$ or optical ${ }^{17}$ means.

\section{References}

1 G. Wulff, Angew. Chem., Int. Ed. Engl., 1995, 34, 1812-1832.

2 P. A. G. Cormack and A. Z. Elorza, J. Chromatogr. B, 2004, 804, 173-182.

3 D. C. Sherrington, Chem. Commun., 1998, 2275-2286.

4 J.-L. Liao, Y. Wang and S. Hjertén, Chromatographia, 1996, 42, 259-262.

5 C. Yu and K. Mosbach, J. Chromatogr. A, 2000, 888, 63-67.

6 E. Yilmaz, K. Mosback and K. Haupt, Anal. Commun., 1999, 36, 167-170.

7 H. Y. Yan and K. H. Row, Int. J. Mol. Sci, 2006, 7, 155-178.

8 S. A. Piletsky and A. P. F. Turner, Electroanalysis, 2002, 14, 317-323.

9 S. A. Piletsky, E. V. Piletskaya, T. L. Panasyuk, A. V. El'skaya, R. Levi, I. Karube and G. Wulff, Macromolecules, 1998, 31, 21372140 .

10 O. Oktar, P. Caglar and W. R. Seitz, Sens. Actuators, B, 2005, 104, 179-185.

11 V. M. Mirsky, T. Hirsch, S. A. Piletsky and O. S. Wolfbeis, Angew. Chem., Int. Ed., 1999, 38, 1108-1110.

12 C. Baggiani, G. Giraudi, C. Giovannoli, C. Tozzi and L. Anfossi, Anal. Chim. Acta, 2004, 504, 43-52.

$13 \mathrm{~S}$. Hjertén, personal communication.

14 N. Kirsch, C. Alexander, S. Davies and M. J. Whitcombe, Anal. Chim. Acta, 2004, 504, 63-71.

15 N. Lavignac, K. R Brain and C. J. Allender, Biosens. Bioelectron., 2006, 22, 138-144.

16 A. G. Mayes, J. Blyth, M. Kyrolainen-Reay, R. B. Millington and C. R. Lowe, Anal. Chem., 1999, 71, 3390-3396.

17 B. K. Lavine, N. Kaval, D. J. Westover and L. Oxenford, Anal. Lett., 2006, 39, 1773-1783. 